Satisfaction with access to endoscopy training rose from $50 \%$ to $100 \%$ and satisfaction with communication increased from $66.7 \%$ to $100 \%$.

Conclusion Satisfaction amongst trainees and up-skilling in endoscopy has improved significantly with our intervention.

Our model was devised by trainees for trainees. This could be implemented across all trusts and future regional academies to improve access to endoscopy training.

\section{MEASURING DISRUPTION OF UK GENERAL PRACTICE BY DIGITAL FIRST PRIMARY CARE: A CASE STUDY EXAMINING THE IMPACT OF BABYLON GP AT HAND IN LONDON}

Saqib Zakaria Latif, Jonathan Turnbull-Ross, Hui Wang, Sudip Chowdhury. Alliance Manchester Business School, UK

\subsection{6/leader-2021-FMLM.42}

Background General Practice is central to the NHS but remains difficult to access for the working well. It has resisted organisational change leaving it resistant to technology and vulnerable to competition. Privately funded Babylon GP at Hand (BGPaH) can disrupt through a value proposition (access) whilst undermining core funding.

Aim It is important to analyse the impact that Babylon has made in attracting London based patients. In 2017 Babylon created a joint venture with a London based practice resulting in list growth to the 5th largest in the UK. The study examines factors that make General Practice vulnerable to digital transformation.

Design and Setting Adopting a qualitative case-study approach we assessed GP awareness of technological disruption and their willingness to utilise scale-economics through organisational development. The setting of our research was London. Quantitatively, we investigated patient-flow within Hammersmith and Fulham CCG.

Method We utilised a mixed-methods approach utilising semistructured interviews and analysis of public data. Six GPs and two senior health managers were interviewed $(n=8)$ and then analysed using thematic analysis. We also accessed the NHS Business Services Authority Database to compare numbers for each practice in Hammersmith and Fulham on November 2017 and July 2020.

Results Five broad themes were identified including GP Business Ideation, GP Organisational Development, Economies of Scale, COVID-19, and the NHS. The quantitative aspects of the study demonstrated a statistically significant increase in patient number by BGPaH.

Conclusion GPs exhibited an awareness of business threat from $\mathrm{BGPaH}$ but this did not translate to organic organisational change. Factors included the pandemic and the use of technology as well as the emergence of PCNs. There was evidence of successful use of economies of scale by a GP-owned Federation. We could not quantitatively prove that $\mathrm{BGPaH}$ had disrupted primary care.

\section{A NEW STUDENT LED LEADERSHIP PROGRAMME AT WARWICK MEDICAL SCHOOL}

Julia Jones, Charlotte Caroff, Anne-Marie Chilton. Warwick Medical School

10.1136/leader-2021-FMLM.43
This year marks the completion of the inaugural Warwick Medical School Leadership programme. The programme was set up to meet student interest and the need for an effective medical student leadership curriculum highlighted by the UK General Medical Council, the NHS Leadership Academy and The King's Fund.

The programme's aim is to help produce valued future leaders of the NHS. It focuses on creating an engaging and inclusive curriculum for time poor students from a variety of backgrounds.

To achieve this aim, we needed to not only give our students a foundation in leadership skills but empower and inspire them to continue developing these skills outside the course. We devised 5 components to the course, not only to teach the basics but also to support students in building a network of resources and leadership mentors. Students were given tasks to use these tools to understand their benefits for their own ongoing projects .

Within each of the course's assessments students showed an understanding and enthusiasm for the course. Comparing their initial 'project plan' assessment and the final summative reflection, all students demonstrated a developing understanding of leadership roles, greater personal insight and the desire and confidence to develop as a leader. Within the summative reflection, all students stated the course had a positive impact on their current leadership roles

Within the end of course feedback, when asked what they found helpful about the course, students described developing a support network of peers and mentors, interesting and thought provoking seminars and a new confidence to take on new leadership positions and projects.

We are very pleased with how the first year has been received by the students. We recommend other universities encourage similar courses to run. The course has so far proven self-sustaining, with its students developing the skills required to continue its development and the enthusiasm to take over its leadership.

\section{NATIONAL LEARNER ASSEMBLY FOR EQUALITY, DIVERSITY AND INCLUSION}

Gregory FW Stamp, Raees Lunat, Vidushi Golash. Health Education UK

\subsection{6/leader-2021-FMLM.44}

HEE's Summer 2020 call for evidence answered by over 400 trainees the aftermath of Black Lives Matter. Analysis of these responses showed system-wide challenges that trainees face. Those of minority backgrounds and International Medical Graduates are acutely aware of differential attainment and a lack of representative role-modelling. One stand-out issue was difficulties in having trainee views and experiences represented to senior leaders of HEE in open dialogue. This Assembly aims to improve this situation.

Our inaugural event was 26th May 2021. It was attended by Dr Navina Evans and Prof. Namita Kumar, outlining their experiences of the importance of diverse leadership.

An interactive roundtable discussion with over 70 participants followed. Trainees' personal stories were heard and reflected upon. A range of themes emerged, including EDI in recruitment processes, clinical supervision and flexible training options. Some individuals shared experiences of differential attainment, systemic racism and undermining behaviours. 
Actions required

1. Clear guidance at induction on how to escalate concerns both within the employer and the local office.

2. That naming or investigating a concern under a 'Dignity at Work Policy' does not immediately reassure the individual that their complaint of bullying, undermining or discrimination will be recognised as such.

3. Ensure timely investigation and communications.

4. Differences between Revalidation and ARCP processes made clear to doctors in training.

5. Visa sponsorship rules on salary thresholds may preclude some doctors training LTFT.

Importantly, examples of good practice and positive experiences were highlighted. Overall, attendees were supportive of each other, exchanging challenges faced and how they have overcome them, creating interpersonal camaraderie. We anticipate continuing to work together to make beneficial change through future Assemblies.

\section{NATIONAL VITAMIN A PLUS CAMPAIGN (NVAC), 2020 DURING COVID-19 PANDEMIC SITUATION: EXPERIENCE FROM BANGLADESH}

Tasnim Rahman Disu*, Vitamin A Plus Cell, IPHN. Institution of Public Health Nutrition, Dhaka, Bangladesh

\subsection{6/leader-2021-FMLM.45}

Aims National Vitamin A Plus Campaign (NVAC), 2020 which is a Government Program of Bangladesh was implemented to reduce Childhood blindness, Vitamin A deficiency and boosting immunity along with enhanced leadership skills during COVID-19 pandemic situation.

Methods A Mixed method study was implemented aiming to provide intervention with blue colored vitamin A capsules to children aged between 6 to 11 months and red capsules to children aged between 12 to 59 months covering 120,00 distribution centers (permanent) in entire Bangladesh for two weeks (4-17) October, 2020. Supplementation was given by trained health workers and volunteers. RTMR (Real-Time Monitoring \& Reporting) was used for the NVAC, 2020 to report using cellular devices during campaign operations which was observational in nature. Around 14,000 data were collected. SPSS Version 22.0 was used for analysis.

Results Despite the challenges presented by COVID-19, NVAC, 2020 reached 97 per cent of the target population. During NVAC 2020, 2.53 million children aged between 6 to 11 months and 19.5 million children aged between 12 to 59 months has been provided covering 120,00 distribution centers in Bangladesh. Around 1450 monitors from central level visited almost 14,000 distribution centers while the NVAC was ongoing. Protective measures during COVID-19 were taken appropriately by the volunteers and health workers. The study showed a successful situational leadership and management in healthcare system as well as awareness of community level people though there was a fear of unknown due to COVID19.

Conclusions Vitamin A capsule supplementation is not only for blindness prevention but also for boosting overall immunity in children. So the approach of NVAC 2020 may guide us towards more successful health interventional programs leading to a better future with more empowered skilled leadership.
NVAC; Childhood Blindness; Leadership; COVID-19 pandemic situation;

\section{NAVIGATING THE STORM: STRUCTURING AND SUPPORTING JUNIOR DOCTOR WELLBEING DURING THE COVID-19 PANDEMIC}

Avneet Shahi, Alyssia Broomfield. Hampshire Hospitals Foundation Trust, UK

\subsection{6/leader-2021-FMLM.46}

The COVID-19 pandemic has had profound consequences for junior doctors. At a foundation trust with 2 district general hospital sites, we initiated a multi-faceted programme of support for 390 junior doctors. This was led by the Chief Residents (CR) utilising senior management, clinical psychologists, mess committees and communications team. The programme was inclusive, with a focus on the Foundation Year 1 (FY1) cohort. Junior doctors are often the least experienced of the medical team; the second wave presented a unique challenge for FY1s- who had only ever practised within a pandemic. Weekly CR meetings were held and feedback sought pre- and post- intervention. Initiatives targeted four areas: information, support, social and achievement recognition. Interventions included: evening Junior Doctor COVID-19 updates; prioritisation of the annual Junior Doctor Awards (JDAs); onceweekly bitesize, non-urgent updates instead of anxiety-provoking multiple daily emails; FY1s' support cafés, online mess events and enhanced support through evening sessions with clinical psychologists. COVID-19 evening updates were popular, with a peak of 84 attendees. Feedback was encouraging, indicating a demand for these to continue post-pandemic. The clinical psychologists were well-received with informal feedback praising the sessions. FY1s' support cafés, unfortunately, were hampered by poor turnout during the day. The JDAs received a record number of nominations locally (over 200), and feedback indicated that doctors felt, for the first time in many months, valued and appreciated. Priorities now, are physician burnout and junior doctor attrition: innovative approaches are needed. To provide sensitive support, timing of opportunities must be carefully considered; junior doctors can rarely be released from clinical duties to attend events during working hours. Wellbeing support must be delivered alongside sufficient practical assistance otherwise interventions risk insincerity.

\section{NURSE LEADERSHIP DURING PANDEMIC IN TERTIARY CARE HOSPITALS OF GULBARGA CITY, INDIA.}

Nilofer Naaz*, Shantkumar Nigudgi, Pallavi V T, Shreeshail G, Della. Department of Community Medicine, M.R Medical College, Gulbarga, INDIA

\subsection{6/leader-2021-FMLM.47}

Background This study across two leading hospitals of the City will help in understanding the functioning of nursing care and its importance during crisis like Covid-19 pandemic. It will help us to ascertain the level of preparedness of the healthcare facilities to tackle any future emergencies as well. As Gulbarga was one of the first few cities in the Karnataka state of India during the second wave of Covid-19 to cope sooner and better in comparison to others, a post Covid 